\title{
Fuzzy Optimal Control of Mechanical Systems
}

\author{
Pavel Steinbauer, Michael Valášek
}

Department of Mechanics, Faculty of Mechanical Engineering, Czech Technical University in Prague

Karlovo nám. 13, Prague 2, 121 35, Czech Republic, Tel.: $++420-2-24357491$, Address, country, telephone and fax numbers and e-mail address

\begin{abstract}
This paper describes unique method of fuzzy controller's design, which overcomes the existing problems of design subjectiveness. Algorithm, applications and results are described.
\end{abstract}

\section{Keywords \\ Fuzzy Control, Optimal Control, Non-linear systems}

\section{INTRODUCTION}

Fuzzy control has been becoming a specific application of Fuzzy Logic Theory. As a branch of artificial intelligence it becomes very popular during last years. There are many applications in very different branches (cameras, wash machines, control of processes). Fuzzy Control even became trendy technology, by many people considered as cure-all. Possible reasons are:

- Common knowledge about controlled system is utilised. General knowledge about control strategies (operator's knowledge) is often available as well and can be easily transformed into the formulation of fuzzy controller. Multivalued logic suits well to human representation of knowledge. Nothing is simply black or simply white for us, but more or less black, or grey. 
- Exact mathematical model is not required for design of fuzzy controller. As it is very difficult or even impossible to identify many controlled process or systems, fuzzy control can be taken into account.

- Theory of Fuzzy Logic has been widely developed and proved. First publications are available from seventies and many researchers have been working on proving all the aspects of this field.

Unfortunately this cannot be said about fuzzy control. Many control researchers hesitate whether introduce fuzzy control into their projects. Main objections can be summarised as follows: Many "revolutionary" applications of fuzzy control can be solved by conventional strategies as well. Unfortunately many fuzzy researchers have only designed their fuzzy controllers to successfully imitate some kind of conventional controller and measured success of their work by fitting of FC's behaviour to conventional controller's one. Furthermore, there are no general criteria of stability, there are still no systematic strategies for design of fuzzy control systems. Finally, there is still no methodology, no "operating instructions".

On the other hand fuzzy control enables us to efficiently approximate non-linear control laws by the simple choice of only several parameters. Control or optimal control of non-linear systems that really takes into account the non-linear nature of the plant in the form of the state feedback is very difficult to be designed by traditional means.

This paper is devoted to the solution of a problem whether fuzzy control could be objectively designed generally for non-linear state optimal controller.

\section{INITIAL IDEA}

Fuzzy controller (FC) can be considered as real-time working expert system or non-linear control system (Driankov, 1993), (Vysoký, 1996). We can look at any fuzzy controller from both these points of view, as two basic levels can be distinguished in FC. First one operates with symbolical tools like if-then rules, qualitative fuzzy variables and values, etc. Fuzzy controller differs from mainstream expert systems by the fact, that these structures have to be compiled into the very elementary numerical objects and algorithms to ensure fast, real-time performance.

Most of the available papers operate during design of FC from expert-system point of view. Approach which will be described in this paper is based mainly on the second point of view: Fuzzy control technique can be used for rapid design of highly non-linear controller, which has a lot of parameters. These parameters can be tuned, set up, chosen to meet performance requirements. Fuzzy algorithm is used just for realisation of specific, non-linear controller here.

It has been proved that fuzzy system can approximate, realise any function as exactly as needed (Kosko, 1992), (Klement, 1995), (Bauer, 1994). So any control law, any control function, including that one, which realise optimal control can be made by fuzzy controller as well.

Problem can be formulated as follows: 
For dynamic system:

$$
\begin{aligned}
& \dot{x}=f(x, u) \\
& y=h(x, u)
\end{aligned}
$$

and criterion function:

$$
J(x(t), u(t))=\int_{0}^{T k} g\left(x^{\prime}, x, u, t\right) d t
$$

we search for control function

$$
u=f(x) \text { or } u=f(y)
$$

which will lead the system (1) from some initial state $\Psi$ into the set of required states $\Gamma$ and will fulfil following condition:

$$
\min \{J(x(t), u(t) \mid x \in X, u \in U\}
$$

where $\mathbf{X}$ and $\mathbf{U}$ are ranges of state variables and action variables, $\mathbf{x}$ and $\mathbf{u}$ are vectors of state and action variables. There is an important assumption that the system (1) can be controlled by the controller of the form (3), i.e., the linearization of the system (1) around working point describes the system behaviour in some neighbourhood of this working point.

In conventional control theory we are able to compute optimal control function only for very limited subset of systems, especially for the most simple linear systems.

If we were able to determine control function (3) by means of some optimisation routine, it would be significant progress. It seems to us, that fuzzy control function is very useful for this purpose because of many "tunable" parameters.

\section{NEW METHODOLOGY}

Fuzzy controllers are able to approximate any mathematical function as exactly as needed. This feature enables us to rapidly develop the smooth non-linear control functions with arbitrary shape. However, there is still problem how to determine parameters of the fuzzy function. We have developed the following new methodology of the fuzzy control synthesis:

1. Good non-linear simulation model of the controlled system is developed.

2. Controller inputs (system outputs) important for control are chosen. Their ranges have to be determined. 
3. Structure of the fuzzy controller based on Sugeno approach is defined so that it enables realisation of arbitrary control function with only few parameters. Input fuzzy sets are defined to cover whole input parameter space. A complete set of fuzzy rules [Driankov] is developed and each rule antecedent is assigned to unique output singleton. These singletons are tuned parameters. Rule base can be also modified so that the groups of neighbouring rules are assigned with the same singleton. As complete rule bases for fuzzy controller with many inputs extensively enlarge, this technics of "rule grouping" can significantly decrease number of tuned parameters without the loose of fuzzy controller's generality.

4. In case of need of highly non-linear control the fuzzy controller is prepared according to Dynamic Programming Principle (Štecha 1985). The input space of the controller is divided into several tied "zones" and the control law is tuned just in one zone which enlarges the result of previous zones.

5. Then we may design parameters of the controller for each zone separately, either by some conventional, known method (LQR, pole placement for linearized system about the steady state) or by optimisation or by combination of both approaches.

6. The initial conditions of the controlled system are chosen so that outputs (or states) do not exceed the considered zone.

7. Sophisticated multi-parameter global optimisation method is then applied to the output singleton's tuning. No a priori information is needed in case of, at least, marginally stable plant, however some starting setting of the fuzzy controller can be very helpful and can significantly decrease time of optimisation.

8. In case of need to control unstable system, we fall into the problems. The value of the objective function (2) reaches infinity or very high values immediately and that is the reason why objective function is not sufficiently sensitive to the changes of fuzzy controller and the optimisation process does not converge. So we must overcome it by determination of the stabilising fuzzy controller before optimisation is started. This is performed in several steps:

i. First of all, linearized model in the state description [A, B, C, D], based on the non-linear model of the plant is created. As linearization of the plant is not usually simple and straightforward, in particular for systems modelled by qualitative model, we use numerical linearization based on the work (Taylor, 1992).

ii. Although the obtained linearized model is not exact in many cases, it suffices for design of stabilising feedback loop by means of Linear Control Theory. This control law also stabilises the original nonlinear plant around the point of linearization.

iii. The developed linear feedback control law is then transformed by special algorithm into the fuzzy controller structure so that fuzzy controller has the same properties and characteristics. 
iv. Such a fuzzy controller provides good initial values of tuned parameters and, at the same time, ensures sufficiently sensitive objective function for optimisation, as proposed in steps 7,8 .

\section{METHODS OF OPTIMISATION}

First we had to choose parameters of fuzzy controller to be optimized. Scaling factors, input fuzzy sets, number of them, output singletons, rule base, inference algorithm, fuzzy logic operators are available. We decided to optimise output singletons without any significant restriction of generality of the fuzzy controller (see also chapter 3, point 3).

We tested several optimisation algorithms, both gradient and non-gradient: Quasi-Newton method, Rosenbrock method, Simplex method (Rao, 1978), (Lederer, 1988). As fuzzy controller is non-continuous, non-smooth function, gradient method failed. The modification of Powell's method has been also tested. Good result has been obtained by old simplex method (MATLAB function fmins). However, simplex method is only local optimisation method. We need robust, global optimisation method.

As the results of MATLAB Optimization Toolbox (Grace, 1995) were very poor and it lacks global methods, we have enhanced and quite successfully applied univariate search method. We call it discrete search method. Parameter space is searched along the parameter axes so that considered interval is discretized, for each point on the axis the value of objective functions is computed (the other parameters are fixed). Then the best (minimum) value of the objective function and parameter value are chosen and the search continues on the next parameter axis perpendicularly. New parameter axis is discretized, all the other parameters are fixed and new parameter is altered. This approach enables us to overcome local minimum.

Further we developed bridge - interface from the MATLAB, our basic application environment, to the powerful optimization package UFO (Lukšan, 1997 ) as it provides wide range of optimisation procedures including global ones and previous results of this package were very promising in comparison with other optimization tools (e.g. already mentioned Optimization Toolbox for MATLAB).

The fuzzy control function has been also optimized per zone of whole state space according to the principles of Dynamic Programming.

\section{REALIZATION AND IMPLEMENTATION}

Efficient MATLAB/SIMULINK environment was used for models development and verification and also for calculations because of simple handling with nonlinear systems, rapid design of simulation models and fast optimization routines design, prototyping. Structures of fuzzy controller have been implemented in Fuzzy Logic Toolbox (Roger, 1995). It enables us to easily transform our developed controller directly into code of DSP card used for real model control. 


\section{APPLICATIONS}

After successful development of the fuzzy controller for simple 2nd order linear system we also successfully applied this method on semi-active control of truck's suspension. Highly non-linear quarter car simulation model of the 4th order was used. We choose fuzzy state feedback, it means fuzzy controller had 4 inputs and 1 output. That caused very large rule base and many parameters to be optimized.

The second implementation was inverted pendulum. Both linear and non-linear simulation models were developed, checked and real experiment is now being built (Figure 1). In this case we developed fuzzy controller based on LQR for linearized model.

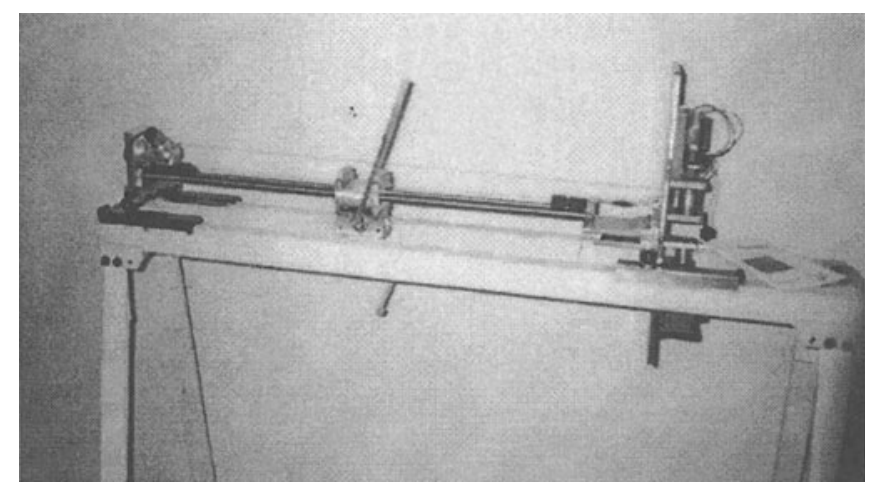

Figure 1 - Controlled inverted pendulum model

Optimization of scaling factors causes instabilities in optimization so we decided to set up them by hand. It is not real restriction, because ranges of input and output variables are usually known. We developed algorithm for optimization which does not really rely on initial starting guess of tuned parameters for tested system. However other more robust, global optimization methods should be explored.

Figure 2 shows results of fuzzy LQR control of inverted pendulum model. These results are almost the same as results of pure LQR control. The performance criteria's values (time integral of dynamical forces to the road) of fuzzy controlled semi-active damper and passive damper applied on 1/4 car model are shown on the figure 3 together with road profile. It can be seen, that performance criterion has been significantly decreased. 


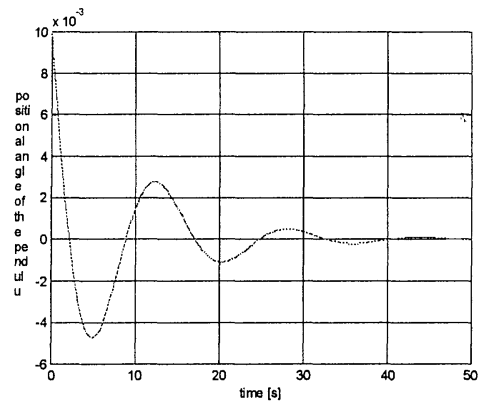

Figure 2 - Control of inverted pendulum by Fuzzy LQR

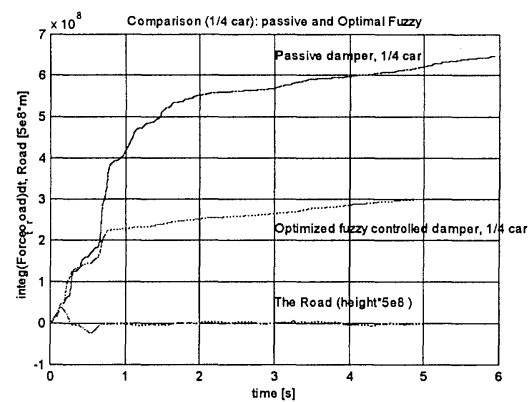

Figure 3 - Performance criteria of fuzzy controlled semi-active damper

\section{CONCLUSIONS}

The results seem to be promising and very interesting, because they could enable us to find optimal control for non-linear systems and systems without mathematical numerical models.

Many previous papers dealing with this topic have required the initial control for optimization routine to be already stable. This restriction has been overcome in our algorithm. This approach also leads towards systematic and objective design procedure opposite to present practice of highly subjective design of fuzzy control.

In the future we are going to expand and explore our method in several directions:

- Problems with optimization of controllers in conjunction with large systems should be overcome (many inputs to the controller increase number of tuned parameters heavily).

- New, better optimization algorithm will be extensively explored. In the concrete, global optimization methods as well as dynamical systems optimization methods, especially those implemented in UFO package are being applied.

- More real model tests should be done.

\section{REFERENCES}

Bauer, P., Klement, E. P., Moser, B. (1994) Modelling of Control Functions by Fuzzy Controllers. JKU Linz.

Driankov, D., Hellendoorn, H., Reinfrank, M. (1993) An Introduction to Fuzzy Control. Springer-Verlag. New York.

Grace, A. (1995) Optimization Toolbox, User's guide. The Mathworks, Inc.

Jang Roger, J.-S., Gulley, N. (1995) Fuzzy Logic Toolbox, User's guide. The

Mathworks, Inc. 
Klement, E. P., Koczy, Moser, B (1995) Are Fuzzy Systems Universal Approximaters? JKU Linz.

Kosko, B. (1992) Neural Networks and Fuzzy Systems. Englewood Cliffs. Prentice Hall.

Lederer, P. (1988) Teorie a optimalizace mechanických systémù. ÈVUT FSI. In Czech.

Lukšan, L., Tùma, M., Šiška, M., Vlèek, J., Ramešová, N. (1997) "Interactive System for Universal Functional Optimization (UFO) - Version 1997", Technical Report No. V-738, December 1997

Rao, S. S. (1978) OPTIMIZATION, Theory and Applications. Wiley Eastern Limited. Bombay.

Taylor, J.H. and Antoniotti, A. J. (1992)"Linearization Algorithm for Computer Aided Control Engineering", Presented at the 1992 IEEE Symposium on Computer Aided Control System Design, Napa, CA, March 17-19, 1992.

Vysoký, P (1996). Fuzzy øízení. ĖVUT FEL. Praha. In Czech.

Štecha, J., Horáèek, P. (1985) Optimální øídící systémy. Fakulta elektrotechnická, ÈVUT. In Czech.

\section{BIOGRAPHY}

Pavel Steinbauer, born in Prague, is PhD student in CTU-FME in Prague. His thesis are dealing with this topic of fuzzy control. He spent 3 months in Fuzzy Logic Laboratorium Linz in JKU, where he joined Fuzzy Logic Workplace development team. Furthermore, he joined team working on the project SemiActive Damping of Truck Suspension.

Michael Valášek is Professor of Mechanics at the Department of Mechanics, Faculty of Mechanical Engineering, CTU in Prague. The author of four textbooks and more than 250 professional papers, he is a member of the International Federation for the Theory of Machines and Mechanisms Technical Committee on Mechatronics. Prof. Valášek received the Ph.D. degree (1984) in control engineering and the Dr.Sc. degree (1991) in computer-aided design from the CTU in Prague. His scientific activities are mainly devoted in computational mechanics of the multibody systems, control, especially control of the non-linear mechanical systems and robots, mechatronics, expert systems. 